\title{
Macht der NGO über die Unternehmen : Druck, Partnerschaft, Evaluation
}

Antoine Mach

\section{(2) OpenEdition \\ 12 Journals}

\section{Electronic version}

URL: http://journals.openedition.org/sjep/883

DOI: 10.4000/sjep.883

ISSN: 1663-9677

\section{Publisher}

Institut de hautes études internationales et du développement

\section{Printed version}

Date of publication: 1 mai 2002

Number of pages: 109-129

ISSN: 1660-5926

\section{Electronic reference}

Antoine Mach, « Macht der NGO über die Unternehmen : Druck, Partnerschaft, Evaluation »,

Schweizerisches Jahrbuch für Entwicklungspolitik [Online], 21 | 2002, Online erschienen am: 10

September 2012, abgerufen am 07 September 2020. URL : http://journals.openedition.org/sjep/883 ;

DOI : https://doi.org/10.4000/sjep.883 


\title{
MACHT DER NGO ÜBER DIE UNTERNEHMEN: DRUCK, PARTNERSCHAFT, EVALUATION
}

\author{
ANTOINE $\mathrm{MACH}^{*}$
}

\section{EINFÜHRUNG}

Die Globalisierung der Wirtschaft und die Konsequenzen des «Shareholder value»-Modells weckten Besorgnis, auf welche die Unternehmen mit Initiativen in der sozialen Verantwortung reagieren. Die Diskurse und Aktionen sollen der Öffentlichkeit beweisen, dass die Unternehmen zwar vorrangig darauf aus sind, für die Aktionäre Gewinne zu erzielen - um den Preis von Standortverlagerungen und Entlassungen - aber auch über den Kreis ihrer Kunden und Mitarbeiter hinaus zum Wohlergehen der breiten Bevölkerung beitragen. Die Nichtregierungsorganisationen (NGO) spielen in diesem Prozess eine wichtige Rolle. Zum einen sensibilisieren sie die Öffentlichkeit mit Demonstrationen und Druckkampagnen, welche die Regierungen und immer direkter die Unternehmen ins Visier nehmen, und zum anderen tragen sie zusammen mit bestimmten Unternehmen im Rahmen von Partnerschaften zur Verbesserung der Arbeitsbedingungen in den Produzentenländern oder zur Korruptionsbekämpfung bei.

Gezielte Kampagnen und Partnerschaften können als direkte Beziehungen zwischen NGO und Unternehmen betrachtet werden, wobei letztere ausserdem in Entscheidungsprozessen im politisch-institutionellen Bereich indirekte Beziehungen unterhalten. Im vorliegenden Artikel geht es um die erste Kategorie. Dieser Typ von Beziehungen wurde als Aspekt der zivilen - getrennt von der legalen - Regelung der Gesellschaft (Bendell, 2000), als Selbstregulierung der Unternehmen (Utting, 2001), als Ausdruck eines globalen Rechts ohne Staat und als private Governance-Systeme (Teubner, 1997) beschrieben. Zurzeit ist eine Diskussion zur politischen Wirksamkeit der zivilen Regelung und zu den realen sozialen Veränderungen, welche von den Druckkampagnen und Partnerschaften zwischen NGO und Unternehmen sowie von den an solchen Mechanismen orientierten öffentlichen Anreizpolitiken zu erwarten sind, im Gange. Einige Kreise fordern, künftig die Forschungsarbeit und die Sensibilisierung der Öffentlichkeit auf dieses Modell hin auszurichten, die Marktkräfte wirken zu lassen, über Verbraucher und Aktionäre direkt Einfluss auf das Verhalten der Unternehmen $\mathrm{zu}$ nehmen und dem öffentlichen Interesse über die gesetzlichen Auflagen hinaus zu dienen. Andere dagegen bezeichnen die soziale Verantwortung der Unternehmen als Irrweg und Mythos, da sie eine Konvergenz zwischen den Interessen eines Privatunternehmens und jenen der Gemeinschaft als zu unwahrscheinlich beurteilen. Daher plädieren sie für weiter gehende Vorrechte des öffentlichen Sektors und für stärkeren Druck auf nationale und internationale Institutionen, um die gesetzlichen Zwänge für die Wirtschaftsbeteiligten zu verschärfen.

* Politikwissenschaftler und Forschungsverantwortlicher, Covalence AG, Genf. 
Der vorliegende Artikel befasst sich mit den direkten Beziehungen, die seit 1970 zwischen NGO und Unternehmen im Zusammenhang mit rund zwölf sozio-ökonomischen Themen von globaler Tragweite bestehen. Ausserdem umfasst der Artikel eine Zusammenstellung der Beziehungen zwischen NGO und Unternehmen sowie zum Schluss eine Evaluation der politischen Chancen der unterschiedlichen Typen von Beziehungen ${ }^{1}$. Die erörterten Fälle betreffen mehrheitlich Beziehungen zwischen schweizerischen Unternehmen und NGO in Themenbereichen, welche die Entwicklungsländer berühren. Die Themen wurden entsprechend den verfügbaren Informationen ausgewählt, wobei Vielfalt und Repräsentativität (unterschiedliche betroffene Industrien) nach Möglichkeit berücksichtigt wurden, aber kein Anspruch auf Vollständigkeit erhoben wird. Die Ergebnisse beruhen auf öffentlich zugänglichen Informationen (Artikel, Internet-Site) und auf Interviews (10 Unternehmen, 20 NGO).

Eine «NGO» wird als Organisation ohne Erwerbszweck definiert, die sich für Menschenrechte, Entwicklungszusammenarbeit, humanitäre Aktion oder Umweltschutz einsetzt. Unter «multinationalen Unternehmen» sind selbstverständlich die an verschiedenen Finanzplätzen notierten privaten Grosskonzerne mit Aktivitäten in mehreren Dutzend Ländern, aber auch mittelgrosse, national verankerte Unternehmen mit Beziehungen zu Partnern im Ausland zu verstehen.

\section{ERGEBNISSE}

Das Verhältnis zwischen NGO und Unternehmen wurde häufig über das Gegensatzpaar Konfrontation - Partnerschaft beschrieben (vgl. Bendell 2000, Elkington and Fennell 1998, Joerchel 1997). Im vorliegenden Artikel wird von der Dichotomie Druck/Partnerschaft ausgegangen («Druck» ist dem Terminus «Konfrontation» vorzuziehen, da er die bisweilen einseitige Natur des Verhältnisses treffender wiedergibt). Es lassen sich verschiedene Arten von Druck und von Partnerschaften zwischen NGO und Unternehmen unterscheiden:

口 Institutioneller Druck: NGO betreiben in nationalen und internationalen politischen Institutionen Lobbying für zwingende Gesetzeserlasse gegenüber Unternehmen. So kämpfen bestimmte Organisationen im Rahmen der Welthandelsorganisation für die Annahme einer Sozialklausel, welche eine weltweite Verbesserung der Arbeitsbedingungen erlauben sollte. In der UN-Subkommission für Menschenrechte schlagen Mitglieder der Arbeitsgruppe für transnationale Unternehmen die Einführung einer für Unternehmen verbindlichen Menschenrechtsnorm vor ${ }^{2}$. Schliesslich plädierten bestimmte NGO anlässlich der jüngsten Überarbeitung der Leitgrundsätze der OECD für multinationale Konzerne ${ }^{3}$ dafür, diesen gesetzliche Verbindlichkeit zu verleihen ${ }^{4}$.

a Informeller Druck: NGO beteiligen sich an Demonstrationen, an welchen von Seattle über Davos bis Genua der Unmut und das Missfallen an der Glo-

1. Der vorliegende Artikel stützt sich auf die Ergebnisse von im Auftrag der Stiftung Antenna internationale durchgeführten Forschungen (Mach 2001) sowie auf spätere Arbeiten bei Covalence AG, Genf.

2. vgl. CETIM, Les activités des sociétés transnationales et la nécessité de leur encadrement juridique, Actes et conclusions du séminaire de Céligny, 4 et 5 mai 2001, <www.cetim.ch/activ/activfra.htm>.

3. <www.oecd.org/daf/investment/guidelines/indexf.htm>.

4. vgl. Bastienne Joerchel Anhorn, Die OECD-Leitsätze für multinationale Unternehmen, Jahrbuch Schweiz-Dritte Welt 2001, IUED. 
balisierung der Wirtschaft und an der Macht der multinationalen Konzerne laut werden. An solchen Kundgebungen werden Kritik und Forderungen an die Adresse der Regierungen der wichtigsten Industrieländer, der internationalen Organisationen wie WTO, IWF und Weltbank sowie der multinationalen Konzerne gerichtet; sie gehören vor den Hintergrund der so genannten «Anti-Globalisierungs »-Bewegung.

• Gezielter Druck: NGO nehmen mit ihren Kampagnen bestimmte Unternehmen und Verhaltensaspekte ins Visier. Diese NGO verfolgen das Ziel, in den Unternehmen punktuelle Verhaltensänderungen zu bewirken. Dazu setzen sie auf die Medien als Informationsvermittler, auf die Sensibilisierung der Öffentlichkeit und auf die Beteiligung der Verbraucher.

- Partnerschaft mit Schwerpunkt auf der Unternehmenstätigkeit: Zusammenarbeit von NGO und Unternehmen in der Definition und Umsetzung von Sozialprogrammen, die auf der freiwilligen Beteiligung des Privatsektors beruhen: Label für fairen Handel, Verhaltenskodizes, soziale Zertifizierung. Die NGO fördern die von den Unternehmen ergriffenen Initiativen und tragen mit ihrem Fachwissen dazu bei.

- Partnerschaft mit Schwerpunkt auf der NGO-Tätigkeit: Andere Partnerschaften beziehen sich nicht auf die Änderung der Wirtschaftskreisläufe, in die das Unternehmen eingebunden ist, sondern auf die Tätigkeit der NGO : Es handelt sich um Sponsoring- bzw. Marketingprogramme für ein bestimmtes Anliegen (z. B. ein Franken pro Verkaufserlös geht an Sozialprojekte).

Der vorliegende Artikel konzentriert sich aus zwei Gründen auf den gezielten Druck und auf die Partnerschaften mit Schwerpunkt auf der Unternehmenstätigkeit: Diese Art Verhältnis ist weniger bekannt und erforscht als der institutionelle und der informelle Druck und bietet ausserdem den NGO sowie den Akteuren in den Bereichen Menschenrechte und Entwicklung offensichtlich besonders viel versprechende politische Chancen.

\section{FALLSTUDIE: GEZIELTER DRUCK; PARTNERSCHAFTEN MIT SCHWERPUNKT AUF DER UNTERNEHMENSTÄTIGKEIT}

\section{$\square$ Handel mit Säuglingsnahrung}

Seit 1974 greifen bestimmte NGO$^{5}$ die Nahrungsmittelindustrie wegen der Kommerzialisierung von Milchpulver in den Ländern der Dritten Welt an. Den Quellen zufolge werden die Milchpulververmarktung in diesen Ländern an sich, aber auch die Verkaufsmethoden kritisiert. Manche Kreise halten es nicht für legitim, in armen Ländern künstliche Milch zu verkaufen - aus gesundheitlichen und aus ökonomischen Gründen (falsch dosierte oder mit verschmutztem Wasser vermischte Milch gefährdet die Gesundheit der Babys; Muttermilch ist gratis, Milchpulver kostet). In den Berichten wird eine Vielzahl von Unternehmen angesprochen und zitiert (für die Schweiz z.B. Migros ${ }^{6}$ ). Nestlé, weltweiter

5. War on Want, Arbeitsgruppe Dritte Welt Bern, Infact, International Baby Food Action Network, Erklärung von Bern, CANES, usw.

6. International Baby Food Network, Breaking the Rules, 1994, zitiert in Ethical Consumer Critic Database, <www.ethicalconsumer.org, 2000>. 
Marktführer für Pulvermilch, steht deutlich im Fadenkreuz der diesbezüglichen Kritik der NGO. 1981 verabschiedete die Weltgesundheitsorganisation (WHO) den Internationalen Kodex für Vermarktung von Muttermilchersatzprodukten. Nestlé und weitere Produzenten traten in den darauffolgenden Jahren dem Kodex bei und setzten damit der eigenen Werbepraxis verschiedene Grenzen. Die Vorwürfe gegen die Nahrungsmittelindustrie gingen anschliessend zwar zurück, verstummten aber nicht völlig, da die Anwendung des Kodex und die Befolgung durch die Industrie ab und an umstritten war. Seit 1999 veröffentlicht Nestlé regelmässig Berichte über Massnahmen in Bezug auf den WHO-Kodex. Dazu können Partnerschaften mit NGO zählen, die Projekte für sauberes Trinkwasser, angemessene Ernährung, Hygiene und medizinische Infrastrukturen fördern ${ }^{7}$.

\section{$\square$ Arbeitsbedingungen in der Bananenproduktion}

Mitte der 70er Jahre organisierte eine Frauengruppe in Frauenfeld Aktionen zur Sensibilisierung der Öffentlichkeit für die Arbeitsbedingungen in den von multinationalen Konzernen kontrollierten grossen Bananenplantagen. Die Frauen kauften in der Migros Bananen ein und verkauften sie am Supermarktausgang für 20 Rappen mehr weiter. Damit wollten sie veranschaulichen, dass die Verbraucher für einen geringen Aufpreis unter humaneren und besseren sozialen Voraussetzungen produziertes Obst kaufen können. Mit dieser im wesentlichen symbolischen Aktion verhalfen die Bananenfrauen ${ }^{8}$ dem Gedanken des fairen Handels mit zum Durchbruch. 1981 startete die Erklärung von Bern eine Kampagne zu den Themen Hunger und Arbeitsbedingungen in den Ländern der Dritten Welt. Dabei wurden die herrschenden Arbeitsbedingungen in den von westlichen Multis kontrollierten Ananasplantagen auf den Philippinen angeprangert. Ausserdem richtete sich die Kampagne gegen Unternehmen wie Migros, die in der Schweiz Ananas vertreiben. Migros bestritt zunächst die Legitimation der Kampagne, trat jedoch anschliessend mit den Verantwortlichen in den Dialog. 1983 wurde zwischen einigen wichtigen schweizerischen $\mathrm{NGO}^{9}$ und der Migros, die sich selbst bei Del Monte einschaltete, eine Sozialklausel betreffend die Ananasproduktion auf den Philippinen ausgehandelt. Die Sozialklausel Migros - Del Monte gehört zu den Pilotprojekten in der unabhängigen Kontrolle der Anwendung einer privaten Sozialnorm im Welthandel ${ }^{10}$.

\section{$\square$ Kampf gegen die Geldwäscherei}

Überall auf der Welt werden Steuergelder unterschlagen und bei Banken gerade auch in der Schweiz - hinterlegt. Die Kapitalflucht untergräbt die öffentliche Ausgabenpolitik und schadet den Interessen der Bevölkerung im Herkunftsland der Gelder. Aus diesem Grund fordern die NGO die Banken auf, einschlägige Klienten strikt zurückzuweisen. Die 1978 gegründete Vereinigung Aktion Finanzplatz Schweiz setzt Einrichtungen, die als zu lax eingestuft werden, unter Druck. Zusammen mit der Erklärung von Bern veröffentlichte sie

7. Nestlé, Rapport sur les actions entreprises en relation avec le Code international de l'OMS, Nr. 4, April 2000, <www.babymilk.nestle.com>.

8. Titel eines Buches von Ursula Brunner, Verlag Huber, Frauenfeld / Stuttgart / Wien, 1999.

9. Brot für alle, Hilfswerk der Evangelischen Kirchen, mit Unterstützung der Erklärung von Bern, Helvetas, Swissaid und Fastenopfer.

10. Vgl. Christoph Stückelberger und Michel Egger, A social clause with a TNC: The Migros - Del Monte Case, Swiss Coalition News 7, Arbeitsgemeinschaft der Hilfswerke, März 1996, S. 6. 
1981 das Manifest für eine Schweiz ohne Fluchtgelder ${ }^{11}$. In den letzten zwanzig Jahren wurden in der Schweiz weitere Aktionen zur Sensibilisierung der Öffentlichkeit für die Geldwäschereiproblematik durchgeführt. Im November 2000 verabschiedeten rund ein Dutzend international tätige Banken - darunter Credit Suisse Group und UBS - in Partnerschaft mit der NGO Transparency International die so genannten Wolfsberg-Grundsätze, die darauf abzielen, die Geldwäscherei effizienter zu bekämpfen.

\section{$\square$ Zugang zu lebenswichtigen Medikamenten}

Mehrere NGO appellieren an die Pharmaindustrie, sich im öffentlichen Gesundheitssystem der Entwicklungsländer stärker zu profilieren und den Zugang zu Medikamenten zur Behandlung von Infektionskrankheiten zu verbessern. Die NGO fordern die Unternehmen auf, einen Teil ihrer Investitionen zur Erforschung und Entwicklung von Therapien gegen Tropenkrankheiten wie Malaria oder die Schlafkrankheit einzusetzen. Ausserdem engagieren sich die NGO für eine Verbilligung der AIDS-Medikamente. Mit diesen Fragen befassten sich Health Action International seit 1981 und die Erklärung von Bern mit Medicus Mundi von 1987 bis 1997 im Bulletin Med in Switzerland. Ärzte ohne Grenzen startete 1999 eine Kampagne für den Zugang zu wesentlichen Medikamenten. Gleichzeitig gingen verschiedene pharmazeutische Unternehmen Partnerschaften mit den NGO ein: Die Leitsätze für Medikamentenschenkungen wurden von internationalen Organisationen im Bereich der öffentlichen Gesundheit, von NGO wie Ärzte ohne Grenzen, Oxfam und Caritas, vom IKRK (1996) und von den wichtigsten Pharmaunternehmen (1999) ${ }^{12}$ angenommen. Die Novartis-Stiftung für nachhaltige Entwicklung arbeitete mit Organisationen wie Helvetas oder Terre des Hommes - Schweiz in Entwicklungsprojekten zusammen ${ }^{13}$; im Global Forum for Health Research ${ }^{14}$ setzen sich Regierungs- und Nichtregierungsexperten sowie Vertreter des Privatsektors mit Fragen der globalen Gesundheitsforschung auseinander.

\section{$\square$ Verhältnis zum Apartheidregime}

In den 80er Jahren forderten verschiedene $\mathrm{NGO}^{15}$ die schweizerischen Industrie- und Bankenkreise auf, ihre kommerziellen und finanziellen Tätigkeiten im damals vom Apartheidregime beherrschten Südafrika zu stoppen. Wie viele Regierungen und auch die Vereinten Nationen vertraten die NGO die Auffassung, die demokratischen Bestrebungen in Südafrika müssten durch einen Boykott unterstützt werden. Nach Ansicht der NGO hat dieser Boykott schliesslich zum Fall des Apartheidregimes beigetragen. Die betroffenen Unternehmen dagegen hielten es mit der von der Schweizerischen Eidgenossenschaft definierten Politik ${ }^{16}$, wonach Wirtschaftssanktionen kein geeignetes Mittel zum Sturz

11. Das Manifest wurde mitgetragen von Fastenopfer, Helvetas, Hilfswerk der Evangelischen Kirchen, Brot für alle, Swissaid, Terre des Hommes - Schweiz.

12. <www.drugdonations.org >.

13. <www.foundation.novartis.com>.

14. <www.globalforumhealth.org>.

15. Anti-Apartheid-Bewegung der Schweiz, Erklärung von Bern, Fastenopfer, Brot für alle, CANES, Aktion Finanzplatz Schweiz, Verein Kritischer AktionärInnen der Bankgesellschaft, usw.

16. Interdepartementale Gruppe Schweiz - Südafrika, Gestern und heute: Beziehungen Schweiz - Südafrika, Schweizerische Eidgenossenschaft, Bern, Juli 1999. <www.dfe.admin.ch/dynamic/Presse _ Rohstoff/CH_RSA/F/af_sud_1f.htm>. 
der Diktatur seien und die Aussetzung der Wirtschaftsbeziehungen vor allem den ärmsten Menschen schaden, ihre Beibehaltung dagegen Kontakte und eine demokratieförderliche Wirtschaftsentwicklung erlauben würde. Trotz der damaligen Dialogversuche der kirchennahen NGO kam es in dieser Angelegenheit zu keiner Einigung zwischen NGO und Unternehmen ${ }^{17}$.

\section{$\square$ Erdölförderung und Menschenrechte}

Erdölgesellschaften werden regelmässig mit Kritik und Forderungen von NGO zur Menschenrechtslage in den Erdöl produzierenden Ländern konfrontiert. Mitte der 90er Jahre appellierten einige NGO an Shell, die Tätigkeiten in Nigeria einzustellen. Amnesty International ersuchte die Firma - erfolglos darum, sich bei der nigerianischen Regierung dafür einzusetzen, die Hinrichtung von neun Aktivisten der Organisation «Movement for the Survival of Ogoni People», die für Menschenrechte und für die Selbstbestimmung des Ogoni-Volkes kämpft $(1995)^{18}$, nicht zu vollziehen. British Petroleum erntete Kritik wegen Verbindungen der privaten Sicherheitsagentur, welche die Anlagen der Firma überwacht, zu den kolumbianischen Streitkräften. Unocal und Total wurden aufgefordert, ihre Tätigkeiten in Birma wegen der nicht vertretbaren Nähe zum Militärregime zu stoppen (vgl. Avery, 2000). Die betroffenen Unternehmen reagierten unterschiedlich auf die Forderungen. Einige - Shell und BP - sprachen in der Folge die Rolle des Privatsektors in Menschenrechtsfragen in der Unternehmenskommunikation offen an. Shell beispielsweise zitiert einen Bericht von Amnesty International: "Amnesty International in its 6 November 1996 report (Nigeria: Time to end the contempt for human rights) states that: "In its approaches in recent years to Shell and other trans-national companies with significant investments in Nigeria, Amnesty International has appealed to them to acknowledge their responsibility to do all that they can to uphold human rights under the Universal Declaration of Human Rights. Only Shell has done so to date.” ( (In jüngster Vergangenheit appellierte Amnesty International in seinen Kontakten mit Shell und anderen transnationalen Konzernen mit Grossinvestitionen in Nigeria an diese Unternehmen, sich nachdrücklich für die Wahrung der Menschenrechte gemäss der Universalen Menschenrechtserklärung zu engagieren) ${ }^{19}$. Allerdings wurden im Menschenrechtsbereich keine wirklichen Partnerschaften zwischen NGO und Erdölunternehmen geschlossen.

\section{$\square$ Arbeitsbedingungen in der Kaffeeproduktion}

Seit den 70er Jahren finden Verbraucher in Dritte-Welt-Läden in der Schweiz und in Europa Produkte aus dem fairen Handel (Direkteinkäufe bei Genossenschaften, überdurchschnittliche Einkommen, langfristige Verträge). 1987 lancierte die Arbeitsgemeinschaft der Hilfswerke einen Appell für fairen Handel, dem sich 100'000 Bürger anschlossen. Mit diesem Appell war kein direkter Druck auf bestimmte Unternehmen verbunden. Im Jahr 1992 gründeten die wichtigsten schweizerischen NGO nach dem Vorbild der holländischen Pioniere

17. Arbeitsgruppe Südafrika - Terre nouvelle - Protestantische Hilfs- und Missionswerke und Rundtisch Südafrika des Schweizerischen Katholischen Missionsrates, Suisse - Afrique du Sud, Faire mémoire pour assurer le présent, Matran, 14/15 janvier 2000, Mai 2000.

18. Pierre Sané, AI and Economic Actors, May 30, 2001, <www.amnesty.org>.

19. <www.shellnigeria.com/shell/hr_rhs.asp $>$. 
die Stiftung Max Havelaar. Aufgabe der Stiftung ist der Vertrieb von Produkten aus dem fairen Handel in den Grossverteilernetzen ${ }^{20}$. Migros und Coop zeigten bald Interesse an der Vermarktung von Produkten mit diesem Label. Die in Supermärkten verkauften Max-Havelaar-Produkte (Kaffee, Schokolade, Zucker, Honig, Bananen, Tee, Orangensaft, Reis) verzeichnen in der Schweiz und in Europa steigenden Erfolg. Rekordergebnisse unter den europäischen Ländern erzielt die Schweiz mit einem Marktanteil von 3\% für mit dem Max-HavelaarLabel gekennzeichneten Kaffee, gefolgt von den Niederlanden (2,7\%), Dänemark $(1,8 \%)$ und Grossbritannien $(1,5 \%)^{21}$.

\section{$\square$ Arbeitsbedingungen in der Orientteppichfabrikation}

$\mathrm{Ab}$ Ende der 80er Jahre verbreiteten die Medien und internationale NGO Informationen über die Arbeitsbedingungen in der Teppichherstellung in Asien. Das Problem Kinderarbeit liess die Emotionen in der Öffentlichkeit hochschlagen. 1995 schlossen sich Fastenopfer, die Erklärung von Bern, Brot für alle und Swissaid mit der Schweizerischen Interessengemeinschaft Sauberer Orientteppichhandel IGOT zusammen und gründeten gemeinsam das Label STEP ${ }^{22}$. Geschäfte, die sich diesem Label angeschlossen haben, engagieren sich für die Förderung von fairen Arbeitsbedingungen in Produzentenländern wie Indien, Nepal, Pakistan, Marokko oder Iran. Dazu gehört die Verringerung der Kinderarbeit und vor allem die Verbesserung der Lebensbedingungen erwachsener Knüpferinnen und Knüpfer. Vor Ort kontrollieren unabhängige NGO zusammen mit der STEP-Stiftung in der Schweiz, ob die Verpflichtungen aus dem STEPLabel eingehalten werden. Eine vergleichbare Initiative wurde in deutschsprachigen und in angelsächsischen Ländern unter dem Label Rugmark lanciert.

\section{$\square$ Soziale Auswirkungen der grossen Stauwerke}

Im Jahr 1997 lancierten die Erklärung von Bern, die Arbeitsgemeinschaft der Hilfswerke, Greenpeace und der WWF eine Kampagne gegen die Beteiligung der Schweiz am Bau des Drei-Schluchten-Wasserkraftwerks in China ${ }^{23}$. Ähnliche Aktionen wurden im Zusammenhang mit den Stauwerken von Ilisu in der Türkei und Mahesvar in Indien durchgeführt. Laut den NGO wiegen die negativen sozialen und ökologischen Folgen derart schwer, dass die Verwirklichung der Projekte verhindert werden muss. Die Kampagnen der NGO zielen in erster Linie auf die schweizerischen Regierungsstellen und insbesondere auf die Exportrisikogarantie $\mathrm{ab}^{24}$, richten sich jedoch auch gegen einige direkt an den Projekten beteiligte Schweizer Firmen wie ABB, Sulzer oder Credit Suisse Group. Der gezielte Druck der NGO auf die Unternehmen äussert sich in Pressemitteilungen, offenen Briefen, einem Appell für eine Postkartenaktion an Unternehmen und in Wortmeldungen in Aktionärsversammlungen. Vereinzelt konnten sie dabei auf die Unterstützung von institutionellen Anlegern wie der Pensionskasse der Genfer Beamten CIA zählen, welche 1998 in der ABB-Jahresversammlung

20. <www.maxhavelaar.ch>; Max Havelaar Foundation, Swiss Coalition News 5, September 1995.

21. <www.maxhavelaarfrance.org/label4.html>, Ziffern 2000.

22. <www.step-foundation.ch>.

23. <www.ladb.ch>.

24. Stellungnahme des Staatssekretariats für Wirtschaft zur Exportrisikogarantie für das Drei-Schluchten-Wasserkraftwerk in China: <www.admin.ch/cp/37E7A6DE.CB056EF4@seco.admin.ch.html>. 
eine gegen das Drei-Schluchten-Wasserkraftwerk gerichtete Initiative unterstützte. Die mit dem Thema befassten NGO und ABB trafen sich zwar mehrmals zum Dialog, ohne sich jedoch auf Schweizer Ebene auf eine Verständigungsgrundlage zu einigen. Auf internationaler Ebene dagegen wurde 1998 im Rahmen der Weltkommission für Stauwerke - ein auf Initiative der Weltbank geschaffenes Gremium, in dem ABB und die Erklärung von Bern vertreten sind - ein Dia$\log$ zwischen Privatsektor, NGO und Regierungsbehörden ins Leben gerufen ${ }^{25}$. Diese Kommission formuliert Empfehlungen zu den sozialen und ökologischen Auswirkungen der grossen Wasserkraftwerke und widmet sich besonders der Frage der Vertreibung der betroffenen Bevölkerungen und der Gewährung einer entsprechenden Entschädigung.

\section{Tourismus in Birma}

Zahlreiche Beobachter und Institutionen werfen dem Regime in Birma bzw. Myanmar gravierende und wiederholte Menschenrechtsverletzungen vor. Birma ist bei westlichen Touristen ein beliebtes Reiseziel. Deshalb stellt sich die Frage nach den Konsequenzen des Tourismus auf die Menschenrechtslage in Birma. Einige NGO wie die Association Suisse - Birmanie ${ }^{26}$ und der Arbeitskreis Tourismus und Entwicklung (AKTE) ${ }^{27}$ plädieren dafür, Birma als Reiseziel zu boykottieren, weil mit dem Tourismus Kapital ins Land fliesst und damit das Regime stärkt. In der Schweiz führte AKTE im Jahr 1998 eine Kampagne gegen die Migros- und Coop-Reisebüros durch, die Pauschalreisen in Birma organisierten. Der Druck erzielte insofern Wirkung, als Migros und Coop ihre Angebote vom Markt zurücknahmen. In einem Briefwechsel mit AKTE äusserte Migros allerdings Zweifel an der Wirksamkeit von Boykotten für die Förderung der Menschenrechte und der Demokratie. Migros vertritt die Ansicht, dass die Kontakte mit der birmanischen Bevölkerung dank dem Tourismus genau so viel zu einer positiven Entwicklung beitragen können ${ }^{28}$. In diesem Punkt wird Migros unterstützt von Amnesty International - Schweiz und von deren Arbeitsgruppe Wirtschaft und Menschenrechte, die zwar massive Menschenrechtsverletzungen klar verurteilt, aber vor allem auf die Information und Sensibilisierung der Reiseveranstalter für politische Probleme vor Ort setzt, damit Reisende ihr Urlaubsland in Kenntnis der Sachlage auswählen können ${ }^{29}$. In dieser Haltung nimmt Amnesty International - ohne sich grundsätzlich zur Relevanz von Boykotten zu äussern - seit 2000 mit bestimmten Reiseagenturen Kontakt auf und fordert eine umfassende Kundeninformation. Die Menschenrechtslage im Urlaubsland muss der Kundschaft klar dargelegt werden. 1998 wurde von Frankreich aus mit dem Label Tourism for Development eine ähnliche Initiative gestartet: Reisende sollen Hoteliers auswählen können, die zur wirtschaftlichen und sozialen Entwicklung ihres Landes beitragen, indem sie $1 \%$ der Übernachtungseinnahmen an von NGO durchgeführte Projekte abführen ${ }^{30}$. Das Label wird von Luftfahrtgesellschaften und von Reiseagenturen unterstützt.

25. <www.dams.org $>$.

26. <www.birmanie.int.ch/ asb>.

27. <www.akte.ch>.

28. Michel Danthe, Direktor Presseveröffentlichungen Migros, Antwort an Mitglieder von AKTE, 23. Juli 1998, zitiert in Mach 2001.

29. Gespräch mit Danièle Gosteli, Arbeitsgruppe Wirtschaft und Menschenrechte, Amnesty International- Schweiz, 16. November 2001.

30. <www.tourismfordevelopment.com>. 


\section{$\square$ Arbeitsbedingungen in der Bekleidungsindustrie}

1999 lancierten die Erklärung von Bern, Brot für alle und Fastenopfer die Clean Clothes Campaign für gerecht produzierte Kleider. Es handelt sich um eine ursprünglich holländische Initiative, die an eine ähnliche, auf Marken- und Sportschuhhändler ausgerichtete Kampagne (Lets'go fair) anknüpft. In der Clean Clothes Campaign werden Marken- und Bekleidungshändler aufgefordert, sich bei Lieferanten und Zulieferern für die Befolgung der grundlegenden Normen der Internationalen Arbeitsorganisation einschliesslich der Gewerkschaftsrechte stark zu machen. Dazu wurde der Clean Clothes-Kodex entwickelt. Ausserdem sollen die Verbraucher im Rahmen der Kampagne Postkarten an die betroffenen Schweizer Firmen schicken. Über 60'000 Postkarten wurden an Adressaten gesandt, die unterschiedlich reagierten - einige stellten sich taub, andere versprachen Initiativen, welche den Erwartungen der NGO mehr oder minder entsprechen. Die Druckkampagne läuft heute weiter. Drei anvisierte Unternehmen - Migros, Veillon und Switcher - haben eine Partnerschaft mit den NGO abgeschlossen, welche die Clean Clothes Campaign organisierten. Die im Jahr 2000 abgeschlossene Partnerschaft besteht aus einem Pilotprojekt zur unabhängigen Kontrolle der Anwendung des Clean ClothesKodex in den Produzentenländern ${ }^{31}$.

\section{$\square$ Debatte über die Todesstrafe}

Am 7. Januar 2000 startete Benetton eine Werbekampagne vor dem Hintergrund der Vereinigten Staaten: Unter dem Titel «We, on death row» werden Porträtaufnahmen von Todeskandidaten mit Personenangaben vorgestellt. Laut Benetton verfolgt die Kampagne das Ziel, eine Diskussion zur Todesstrafe hervorzurufen und den Insassen der Todeszellen die verloren gegangene Menschlichkeit zurückzugeben: Die «anständigen» Bürger, die sich immer im Recht sehen, sollen wissen, dass es um Männer und Frauen aus Fleisch und Blut geht und nicht um virtuelle Figuren, die man wie in einem Videospiel mit einem Mausklick beseitigen kann ${ }^{32}$. Die National Association of Criminal Defense Lawyers trug zur Kampagne bei. Im Pressecommuniqué vom 10. Januar begrüsst (welcomes) Amnesty International (AI) die Benetton-Initiative, die laut AI eine eingehende Diskussion zur Abschaffung der Todesstrafe herbeiführen könnte ${ }^{33}$. Diese Mitteilung kann als Ausdruck vorsichtiger Unterstützung der Benetton-Kampagne durch Amnesty International, nicht aber als eigentliche Partnerschaft zwischen der NGO und der Firma gesehen werden ${ }^{34}$. In einer am 28. Januar veröffentlichten Pressemitteilung ${ }^{35}$ wird ausserdem präzisiert, dass Amnesty International weder an der Konzeption der Kampagne noch am Beschluss zur Lancierung beteiligt war. AI äussert zudem Mitgefühl mit den Opfern von Gewaltverbrechen und mit ihren Familien und verspricht, gegen alles vorzugehen, was die Ängste dieser Menschen zusätzlich verschärfen könnte. In den

31. <www.cleanclothes.ch>.

32. Benetton, Looking at death in the face, Pressemitteilung, Ponzano, 7. Januar 2000, <www. benetton.com/wws/aboutyou/campinfo/index.html>.

33. Amnesty International's position regarding Benetton's worldwide campaign about the death penalty, 10. Januar 2000, http://www.web.amnesty.org/ai.nsf/index/ACT500022000

34. Aus diesem Grund steht das Symbol in der Darstellung auf der folgenden Seite in Klammern.

35. Press release, Amnesty International's reaction to Benetton's ongoing campaign against the death penalty, 28. Januar 2001, <www.web.amnesty.org/ai.nsf/index/ACT500032000>. 
Vereinigten Staaten löste die Kampagne bei Angehörigen von Opfern und bei Organisationen, welche die Todesstrafe befürworten, lebhaften Protest aus ${ }^{36}$. Vor den Sears-Warenhäusern - wichtige Benetton-Verteiler in den USA - fanden Demonstrationen statt. Am 17. Februar verlautbarte die Geschäftsleitung von Sears, dass in den 400 Warenhäusern in den Vereinigten Staaten keine BenettonArtikel mehr verkauft würden. Im April 2000 stellten 12- bis 14-jährige Schüler in Genf im Bürgerkundeunterricht Fragen zur Todesstrafe; Auslöser der Diskussion waren die Plakate der Benetton-Kampagne. Es folgten eine Klassenarbeit sowie ein Austausch und ein Briefwechsel der Schüler mit amerikanischen Todeskandidaten. Am 2. Mai wurde in Rom der Photograph Oliviero Toscani nach 18 Jahren Zusammenarbeit von Luciano Benetton entlassen.

\section{Abbildung 1: Druck und Partnerschaft zwischen NGO und Unternehmen (1974-2000)}

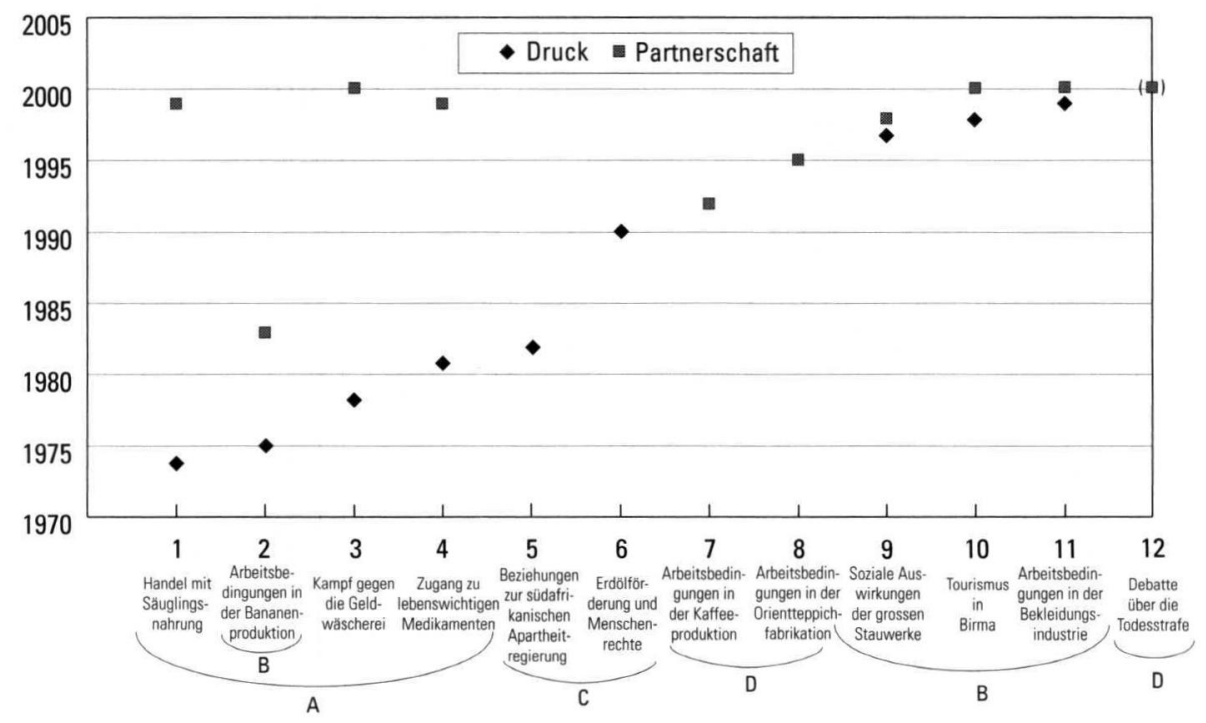

Anmerkung: Die Quadrate und Rauten, die für den Druck bzw. die Partnerschaften stehen, stellen Ausgangspunkte, keine einmaligen Ereignisse, dar: Das jeweilige Jahr markiert den Beginn des Druck- bzw. Partnerschaftsprozesses (z.B. Jahr der Lancierung einer Kampagne, der Unterzeichnung eines Abkommens usw.).

Die 12 Fallbeispiele werden in der Grafik 1 zusammengefasst. Die Grafik bietet einen historischen Überblick über die Druckmomente und Partnerschaften in der Beziehung zwischen NGO und Unternehmen. 7 der 9 Fälle, in denen Druck ausgeübt wurde, führten anschliessend zu einer Partnerschaft - was das Vorurteil widerlegt, wonach NGO und Unternehmen zwangsläufig im Konflikt stünden, und zeigt, dass NGO zur Durchsetzung ihrer Forderungen auch auf Zusammenarbeit mit den Unternehmen setzen. Ausserdem wird das zeitliche Verhältnis von Druck und Partnerschaft ersichtlich, wodurch eine nahe liegende Intuition bestätigt wird: auf Druck folgt eine Partnerschaft. Die Aufteilung der 12 Fälle in 4 Gruppen ermöglicht eine genauere Analyse.

36. vgl. National Organization of Parents of Murdered Children, Pressemitteilung 11. Januar 2000, <www.pomc.com/minealert1.cfm; www.prodeathpenalty.com $>$. 
In diesen Fällen verharrte die Beziehung zwischen NGO und Unternehmen während langer Zeit in der Konfrontationsphase (1974-1981), bevor Partnerschaften ausgehandelt wurden (1998-2000). Allerdings setzen die Partnerschaften dem Druck nicht immer ein Ende, da die als Partner beteiligten NGO nicht unbedingt mit jenen identisch sind, welche die gezielten Kampagnen anführen. Es kommt häufig vor, dass die Unternehmen einerseits den Druck «kritischer NGO » bewältigen und andererseits Partnerschaften mit «kooperativen NGO » aufbauen.

\section{$\square$ Gruppe B (Fälle 2, 9, 10, 11): rasch von einer Partnerschaft gefolgter Druck}

In diesen Fällen folgt die Partnerschaft sehr rasch auf den Druck (Zeitraum 1995-2000). Die Kritikkampagnen führen zwar schnell zu Dialog und Partnerschaft, bestehen jedoch weiter: Die Clean Clothes Campaign (CCC) zum Beispiel umfasst sowohl Druck (auf alle Marken und Kleiderhändler) als auch eine Partnerschaft (3 Unternehmen beteiligen sich am Pilotprojekt zur Anwendung des CC-Kodex). Bemerkenswert ist das Fallbeispiel 2 - Arbeitsbedingungen in der Bananenproduktion -, bei dem bereits Anfang der 80er Jahre auf den Druck hin rasch eine Partnerschaft zu Stande kam. Die Sozialklausel Migros - Del Monte gilt als Vorreiterin für die Partnerschaften mit Schwerpunkt auf der Unternehmenstätigkeit wie die zahlreichen Verhaltenskodizes und Soziallabels aus den 90er Jahren.

\section{$\square$ Gruppe C (Fälle 5 und 6): nur Druck}

Im Gegensatz zu den Entwicklungen in den anderen Fällen kam es in den beiden Beispielen Beziehungen zum Apartheidregime und Rolle der Erdölgesellschaften im Menschenrechtsbereich zu keiner Partnerschaft zwischen NGO und Unternehmen. Bei Boykottaufrufen im Zusammenhang mit der Menschensrechtslage eines Landes wird häufig beobachtet, dass ein Konsens zwischen NGO-Forderungen und Unternehmensposition ausbleibt. Anscheinend fehlt in solchen Situationen eine gemeinsame Interessensbasis zwischen den NGO und den Unternehmen.

\section{$\square$ Gruppe D (Fälle 7, 8 und 12): nur Partnerschaft}

Die Partnerschaft zwischen NGO und Unternehmen wird ohne vorgängigen Druck auf die Unternehmen bzw. auf deren eigene Initiative abgeschlossen, was auf gemeinsame Interessen der Menschenrechtsaktivisten und Wirtschaftsbeteiligten hindeutet. Der Erfolg des Max-Havelaar-Labels für fairen Handel liegt im Interesse der NGO wie der Grossverteiler, die so ohne Gewinnschmälerung das Sortiment erweitern und neue Verbraucher an sich binden können. Ähnlich scheint es im Interesse einer NGO wie Amnesty International zu liegen, dass die Marke Benetton die Todesstrafe zum Thema ihrer Kommunikation macht. Schliesslich wandte sich ein Arbeitgeberverband im Teppichhandel an die NGO, um das Label STEP zu schaffen. Diese Beispiele zeigen, dass die Unternehmen tatsächlich bereit sind, im Menschenrechtsbereich Initiativen $\mathrm{zu}$ ergreifen, solange ihre Situation als private, Wettbewerbsdruck und Rentabilitätszwang unterworfene Einheiten berücksichtigt wird. 


\section{$\square$ Druck der NGO und Sozialbilanz der Unternehmen}

Der vorhergehenden Abbildung ist zu entnehmen, dass in 7 von 9 Fällen auf Druck eine Partnerschaft folgt. Kann angesichts dieser Zahl von echter Macht der NGO über die Unternehmen gesprochen werden? Geht vom gezielten Druck der NGO eine Hebelwirkung auf das Verhalten der Unternehmen aus? Im Mai 2001 wurde die Sozialbilanz für die 28 Unternehmen des Swiss Market Index erstellt ${ }^{37}$. Dabei wurde versucht, die Quantität der Unternehmenskommunikation zur Beschreibung der wirtschaftlichen, sozialen und ökologischen Auswirkungen vor allem auf Schwellenländer und Entwicklungsländer einzuschätzen. Die Resultate fielen stark unterschiedlich aus. Bestimmte Unternehmen liefern eine Fülle von Informationen unter Rubriken wie Nachhaltige Entwicklung oder Unsere soziale Verantwortung, in welchen sie auf die Behandlung der Mitarbeiter, den Schutz der Artenvielfalt, das Engagement zur Verbesserung der Arbeitsbedingungen bei den Lieferanten, die Förderung von Entwicklungsprojekten usw. eingehen. Andere Unternehmen begnügen sich mit der Erwähnung einiger Schenkungen zu wohltätigen Zwecken und des Sponsoring im humanitären Bereich, ohne nennenswerte Informationen über die Unternehmensaktivität preiszugeben. Wie erklären sich die Unterschiede in der Sozialbilanz unter den dreissig grössten Schweizer Unternehmen? Um diese Frage zu beantworten, wurde versucht, eine Korrelation zwischen der Sozialbilanz und verschiedenen Variablen herzustellen. 4 Variablen wurden geprüft: sozialer Druck, Absatzanteile in Entwicklungsländern, Börsenwert und Börsenperformance.

\section{Abbildung 2-5: Erklärung der Unterschiede in der Sozialbilanz Test am Swiss Market Index (Juni 2001)}

\section{Abbildung 2: Sozialbilanz als Funktion des sozialen Drucks}

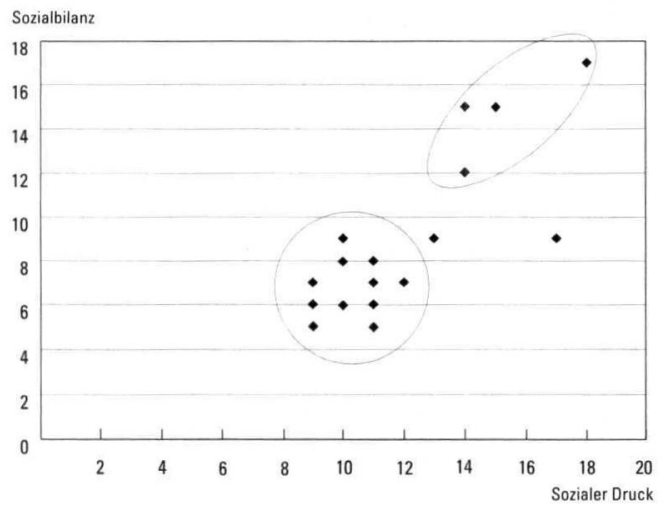

Sozialbilanz: Geschätzte Quantität der vom Unternehmen gelieferten Kommunikation zur Beschreibung des sozialen Impakts.

Sozialer Druck: Geschätzte Quantität der von der Zivilgesellschaft gelieferten Kommunikation (NGO, Universitäten, internationale Organisationen, Medien...) zur Beschreibung des sozialen Unternehmensimpakts.
Sozialbilanz und sozialer Druck stehen in einer positiven Beziehung. Das zeigt, dass die von NGO am stärksten kritisierten und herausgeforderten Unternehmen die solideste Sozialbilanz aufweisen. Dieses Resultat bestätigt die vorhergehende Feststellung und führt einen Schritt weiter: Druck der NGO veranlasst Unternehmen nicht nur zu Partnerschaften, sondern spornt sie individuell zum Handeln an, um die Wahrnehmung und Beurteilung ihrer Sozialperformance zu verbessern. Wenn NGO gezielt Druck ausüben, besitzen sie demnach echte Macht über die Unternehmen. 4 Unternehmen (oben rechts) sind Gegenstand vielfachen Drucks und weisen eine gute Sozialbilanz aus, während sich die Mehrheit der Unternehmen in der umgekehrten Lage befinden: geringer Druck und dürftige Sozialbilanz lunten Mitte).

37. Adecco, ABB, Bâloise, Ciba SC, Clariant, CSG, EMS, Givaudan, Holcim, Julius Baer, Kudelski, Lonza, Nestlé, Novartis, Rentenanstalt, Richemont, Roche, Serono, SGS, Sulzer, Syngenta, Swatch, Swisscom, Swiss Re, Swissair, UBS, Unaxis, Zürich. 


\section{Abbildung 3: Sozialbilanz als Funktion des Absatzmarktes in Entwicklungsländern}

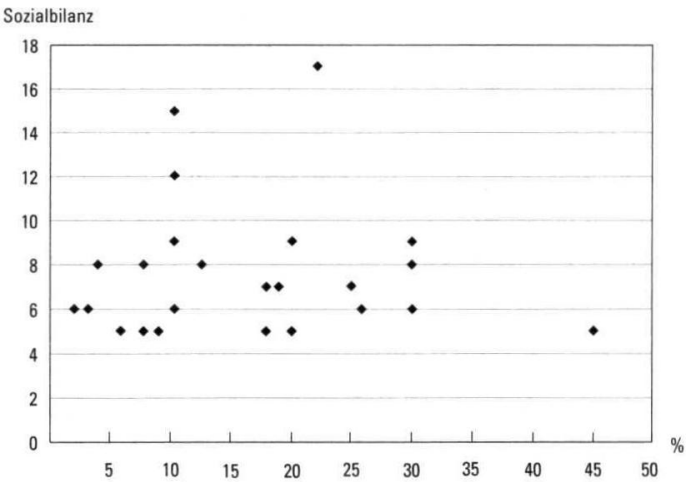

Keine offensichtliche Beziehung zwischen Sozialbilanz und Umsatzzahlen in Entwicklungsländern: Unternehmen mit Absatzmärkten hauptsächlich im Norden ergreifen mehr soziale Initiativen als Unternehmen, welche rund die Hälfte des Umsatzes im Süden erzielen

\section{Abbildung 4: Sozialbilanz als Funktion des Börsenwerts}

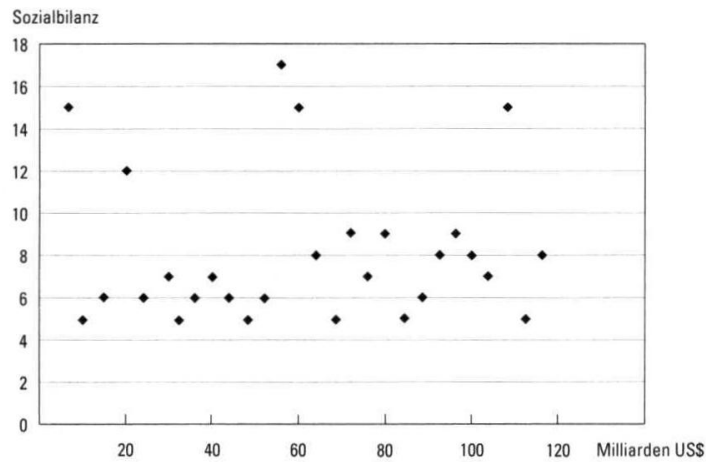

Keine offensichtliche Beziehung zwischen Sozialbilanz und (mit dem Börsenwert ausgedrückter) Unternehmensgrösse: mittlere Unternehmen ergreifen mehr Initiativen als Grossunternehmen.

\section{Abbildung 5: Sozialbilanz als Funktion der Börsenperformance}

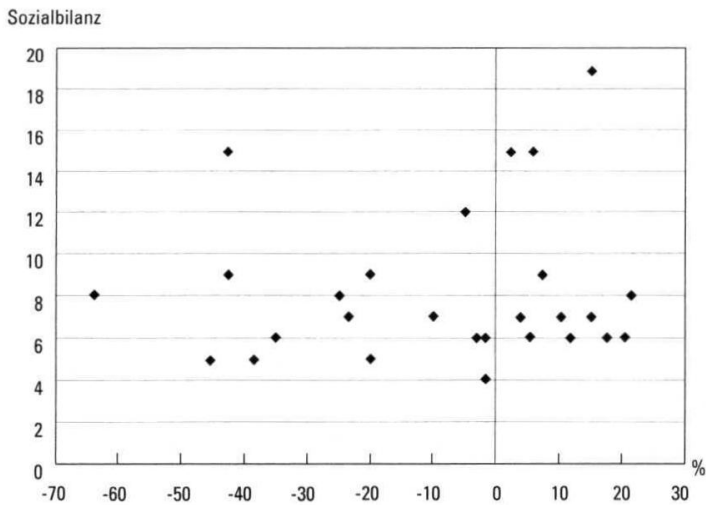

Keine offensichtliche Beziehung zwischen Sozialbilanz und Börsenperformance: Weder lässt sich behaupten, dass finanzielle Rentabilität zu sozialer Performance führt, noch dass soziale Performance finanzielle Rentabilität mit sich bringt.

Die Namen der Unternehmen werden in den Darstellungen nicht ausgewiesen, da diese auf unvollständigen Angaben beruhen und erst provisorische Resultate darstellen. 


\section{$\square$ Einfluss der NGO auf die Beurteilung der Sozialperformance des Unternehmens}

Der Begriff «sozial verantwortliche» oder «ethische Investitionen» taucht im Amerika der 20er Jahre auf, als bestimmte Religionsgemeinschaften beschliessen, ihre Investitionen mit ihren Überzeugungen in Einklang zu bringen. Unternehmen, die Alkohol oder Pornographie verkaufen oder an Gewinnspielen beteiligt sind, kommen für solche Anlagen nicht in Betracht. Eine jüngere Variante der «exklusiven» ethischen Investition bilden die «grünen Fonds», die Ende der 80er Jahre in Europa entstanden und Investitionen in die Automobilindustrie, die Agroindustrie, Kernenergie usw. verbieten. Parallel dazu entwickelte sich ebenfalls auf Betreiben amerikanischer Kirchen mit dem «Aktionismus der Aktionäre» bzw. der «sozial verantwortlichen Kapitalbeteiligung» ein «inklusiver» Ansatz der ethischen Investition. NGO, welche diesen Ansatz verfolgen, kaufen Aktien, um sich in den Jahresversammlungen und bei der Leitung von Unternehmen mit sozialem Verbesserungsbedarf Gehör zu verschaffen (z.B. in der Schweiz CANES mit Nestlé, Verein Kritischer AktionärInnen der Bankgesellschaft, Erklärung von Bern mit ABB, Actares).

Das exklusive ethische Investitionsmodell weist zwei Nachteile auf: In finanzieller Hinsicht verpflichtet es zu einer Verringerung der Risikostreuung und der Gewinnchancen; in ethischer Hinsicht hemmt es die Förderung progressiver sozialer Innovationen bei Industrien, die endgültig als Sünder abgestempelt werden. In den 90er Jahren entwickelt sich unter dem Einfluss des Konzepts der nachhaltigen Entwicklung eine neue Kategorie sozialverantwortlicher Investitionsprodukte: Beim «Klassenbesten»-Modell (best-of-class) werden keine Industrien von vornherein ausgeschlossen, sondern in jedem Sektor die Unternehmen mit dem besten Sozial- und Umweltprofil gelobt. Die gleiche pragmatische Reformhaltung ist bei der aktiven Ausübung der Aktionärsstimmrechte zu beobachten. Produkte, die dieser Tendenz zugeordnet werden, erfordern systematische und nuancierte Auswertungen der Sozialperformance des Unternehmens, die den Banken und Anlegern von Fachinstituten zur Verfügung gestellt werden. In den letzten Jahren wurden nach diesem Ansatz mehrere Nachhaltigkeits-Indizes geschaffen, z.B. der Dow Jones Sustainability Index und FTSE4Good.

Zunehmend interessieren sich auch die konventionellen Finanzakteure für die «nachhaltigen Investitionen» als zeitgenössische Form der ethischen Investitionen. Sie sehen in der Evaluation der Sozialperformance des Unternehmens einen Massstab für die Reputation eines Unternehmens, der wertvolle Hinweise auf mögliche rufschädigende Risiken liefern kann. Diese Entwicklung konsterniert zahlreiche Beobachter im Lager der Ethikbefürworter wie unter den Anlagefachleuten, da beide Kategorien ihre Interessen traditionell als entgegengesetzt betrachteten. Banken wie Sarasin, UBS oder Lombard Odier heuerten Teams von Analysten für nachhaltige Entwicklung an, welche aus Anlegersicht interessante Wertpapiere, aber auch die eigentlichen Banktransaktionen aus dem Blickwinkel der Nachhaltigkeit prüfen. So fordern immer mehr - ethisch oder finanziell motivierte - Investoren ergänzend zur rein ökonomischen Bewertung soziale und ökologische Unternehmensanalysen.

Einige Banken führen die Evaluation der Sozialperformance selbst durch, während andere externe Konsulenten beauftragen. Wie wird der Ethikgrad eines 
multinationalen Konzerns gemessen? Erste Informationsquelle sind die Unternehmen selbst, die Sozial- und Umweltberichte veröffentlichen und ausführliche Fragebögen beantworten. Wie jedoch kann garantiert werden, dass die Antworten der Unternehmen mit der Wirklichkeit übereinstimmen und dass die ausgewiesene Ethik auch praktiziert wird? Welches der weltweit vertretenen Ethikmodelle soll als Referenz dienen? Erfüllen Länderboykotte wegen Menschenrechtsverletzungen wirklich ihren Zweck? Bieten gentechnisch veränderte Organismen (GVO), die NGO und Verbraucher im Norden verurteilen, nicht eine Chance für die Landwirtschaft und die Entwicklung des Südens? Wie steht es mit den grossen Stauwerken? Die Bewertungsstellen erheben nicht den Anspruch, alle Fragen zu beantworten, welche die Experten der ökonomischen und sozialen Entwicklung beschäftigen; sie wenden sich an die Urheber der Ethiknormen, an Regierungen und NGO, an UN-Stellen und Universitäten und bemühen sich, mit den verschiedenen Rückmeldungen - ein Spiegelbild des für unsere moderne (bzw. postmoderne) Gesellschaft bezeichnenden Pluralismus - zurechtzukommen.

NGO beteiligen sich als unfreiwillige Experten indirekt an der Bewertung der Sozialperformance der Unternehmen. Themen wie die sozialen Auswirkungen der grossen Stauwerke, der Kampf gegen die Geldwäscherei, der Handel mit Säuglingsnahrung, GVO oder die Beziehungen mit Birma werden von den Institutionen, die Sozialevaluationen durchführen, bei der Unternehmenseinschätzung berücksichtigt. Auch der Abschluss einer Partnerschaft zwischen einer NGO und einem Unternehmen gilt bei der Evaluation der Sozialperformance des Unternehmens als relevante Information und fällt bei der Benotung durch die Fachinstitute ins Gewicht.

Daneben wirken NGO als freiwillige Experten auch direkt an der Evaluierung der Sozialperformance des Unternehmens mit. Eiris, die in London verwaltete Datenbank zur sozialen Unternehmensverantwortung, Partner der Indizes FTSE4Good, wurde von einem NGO-Netz gegründet. In der Schweiz wurde das Consulting-Büro für nachhaltige Investitionen Infozentrum (Freiburg) ebenfalls von Anhängern der Entwicklungsbewegung geschaffen, sowie unlängst auch Covalence (Genf). Als letztes Beispiel sind eine Reihe von schweizerischen und europäischen Banken zu nennen, die soziale Performance-Indikatoren für die Finanzindustrie entwickeln und dazu die verschiedenen Beteiligten - darunter die NGO - konsultieren ${ }^{38}$.

\section{EVALUATION DER MACHT DER NGO ÜBER DIE UNTERNEHMEN NACH ART DER BEZIEHUNG}

Nach der Analyse der direkten und indirekten Rolle der NGO in der Beurteilung der Sozialperformance des Unternehmens lässt sich die am Anfang des Artikels skizzierte Zusammenstellung der verschiedenen Typen von Beziehungen zwischen NGO und Unternehmen vervollständigen. 


\section{Abbildung 1: Zusammenstellung des Typs von Beziehungen zwischen NGO und Unternehmen: Druck, Partnerschaft, Evaluation}

\begin{tabular}{|c|c|c|c|}
\hline \multicolumn{2}{|c|}{$\begin{array}{l}\text { Typ Beziehung zwischen } \\
\text { NGO und Unternehmen }\end{array}$} & Beispiele & Rolle der NGO \\
\hline \multirow{3}{*}{ Druck } & Institutionell & $\begin{array}{l}\text { Lobbying bei nationalen und } \\
\text { internationalen Institutionen }\end{array}$ & \multirow{3}{*}{ Kritik } \\
\hline & Informell & Demonstrationen von Seattle bis Genua & \\
\hline & Gezielt & Sensibilisierungskampagnen, Boykotte & \\
\hline \multirow[t]{2}{*}{ Partnerschaft } & $\begin{array}{l}\text { Unternehmens- } \\
\text { tätigkeit }\end{array}$ & $\begin{array}{l}\text { Labels, Verhaltenskodizes, } \\
\text { soziale Zertifizierung, Unterstützung } \\
\text { individueller Initiativen }\end{array}$ & $\begin{array}{l}\text { Unterstützung/ } \\
\text { Beratung }\end{array}$ \\
\hline & $\begin{array}{l}\text { Schwerpunkt } \\
\text { NGO-Tätigkeit }\end{array}$ & $\begin{array}{l}\text { Sponsoring, Marketing für } \\
\text { eine bestimmte Sache }\end{array}$ & Unterstützung \\
\hline \multirow{2}{*}{ Evaluation } & Indirekt & $\begin{array}{l}\text { Druckkampagnen und Partnerschaften } \\
\text { zwischen NGO und Unternehmen } \\
\text { werden bei der Evaluation der } \\
\text { Sozialperformance des Unternehmens } \\
\text { berücksichtigt }\end{array}$ & \multirow[t]{2}{*}{ Beratung } \\
\hline & Direkt & $\begin{array}{l}\text { NGO schaffen Organe für die Sozial- } \\
\text { performance der Unternehmen oder } \\
\text { arbeiten mit ihnen zusammen }\end{array}$ & \\
\hline
\end{tabular}

\section{$\square$ Institutioneller Druck}

Im Rahmen der bei den drei genannten Institutionen durchgeführten Projekte Sozialklausel der WTO, Leitsätze der OECD für multinationale Unternehmen, Arbeitsgruppe transnationale Unternehmen der UN-Subkommission für Menschenrechte - soll die Verabschiedung von auch Unternehmen bindenden Menschenrechtsnormen gefördert werden. Die politischen Möglichkeiten sind relativ begrenzt. Gleiches gilt für die Verbindlichkeit gegenüber Staaten in diesem Bereich. Gemäss dem Human Development Report des UNDP von 2000 existieren in der aktuellen Weltordnung nur wenige Schranken, die die Staaten und die globalen Akteure weltweit zur Achtung der Menschenrechte zwingen ${ }^{39}$. In einer von nationalen Souveränitäten geprägten Welt bleiben die Annahme und Umsetzung einer öffentlichen Politik mit globaler Tragweite sehr problematisch. Zu präzisieren ist, dass das Thema institutioneller Druck in der genannten Recherche nur ansatzweise angeschnitten wurde.

Das Verhältnis der Unternehmen zur Menschenrechtsproblematik umfasst zwei Sachverhalte mit unterschiedlichen rechtlichen und politischen Folgen: Achtung und Förderung. Die Unternehmen sind wie alle Organe der Gesellschaft gehal-

39. Human Development Report 2000, Entwicklungsprogramm der Vereinten Nationen, de Boeck-Universtität, 2000. 
ten, die Menschenrechte zu achten (Präambel der Allgemeinen Menschenrechtserklärung) - sprich nicht zu verletzen. Wenn ein Unternehmen in einer Tochtergesellschaft Zwangsarbeit einsetzt, die gewaltsame Niederschlagung einer Gewerkschaftsdemonstration befiehlt oder Menschen gegen ihren Willen als Versuchskaninchen für gefährliche Experimente missbraucht, hat es sich in der Regel vor Gericht zu verantworten. Dafür gibt es einschlägige Gesetzesvorschriften. Daneben werden die Unternehmen auch aufgefordert, die Menschenrechte zu fördern, d.h. sich bei Dritten (Regierungen, Unternehmen und anderen Beteiligten) für die Achtung der Menschenrechte einzusetzen. Darüber hinaus sind die Unternehmen aufgerufen, auch dort zur Verwirklichung der Menschenrechte beizutragen, wo die Dichotomie Achtung / Verletzung bedeutungslos ist, weil insbesondere auch die wirtschaftlichen, sozialen und kulturellen Rechte berührt werden : Recht auf Ernährung, Gesundheit, Unterkunft oder Trinkwasser sind zwar sicherlich unverletzbare Rechte, müssen aber vor allem verwirklicht, umgesetzt und gefördert werden. Daher müssen ein breites Aktionsfeld, eine Vielfalt von Akteuren und womöglich spezifische Umsetzungsregelungen in Betracht gezogen werden, die gewährleisten, dass der Grundsatz der Unteilbarkeit der Menschenrechte nicht verletzt wird.

In der Mehrheit der 12 geprüften Fälle wird das Unternehmen nicht aufgefordert, selbst die Menschenrechte zu achten, sondern bei verschiedenen beteiligten Parteien die Achtung und Verwirklichung der Menschenrechte zu fördern. Das Unternehmen gilt zwar in den Augen des Gesetzes nicht als potenzieller Täter, aber wegen seiner globalen Präsenz wird ihm eine moralische Pflicht zugeschrieben, sich in sozialen und politischen Fragen in entfernten Ländern zu engagieren. Für den Staat und für die Akteure, die Einfluss auf die Regierungspolitik nehmen möchten, eröffnen sich hier viel versprechende Gelegenheiten für Anreizmassnahmen in der sozialen Unternehmensverantwortung. Einige Beispiele: Die Exportrisikogarantie unterbreitet den Bewerbern einen sozioökonomischen Fragebogen; der Bund (seco) unterstützt die Stiftung Max Havelaar seit ihrer Gründung; amerikanische Stadtverwaltungen verabschiedeten öffentliche Beschaffungspolitiken, welche die Unternehmensbilanz in Menschen- und Arbeitsrechten berücksichtigen; britische, australische und (demnächst) deutsche Pensionskassen sind verpflichtet, die Öffentlichkeit über ihre Investitionspolitik zu informieren und nachzuweisen, inwiefern sie ihre Anlagen nach Nachhaltigkeitskriterien tätigen. Ob solche Massnahmen sich zunehmend durchsetzen werden, hängt von zahlreichen Faktoren ab, darunter sicherlich von der Entwicklung der politischen Kultur der massgeblichen Parteien und jener der NGO, Gewerkschaften und Gesellschaftsbewegungen. Wahrscheinlich werden Regierungen und NGO vermehrt zusammenarbeiten mit dem Ziel, die soziale Verantwortung der Unternehmen zu fördern.

\section{$\square$ Informeller Druck}

Dieser Typ von Beziehung wirkt sich offensichtlich nicht unmittelbar auf das Verhalten des Unternehmens aus. Dagegen beeinflusst er möglicherweise andere Arten von Beziehungen zwischen NGO und Unternehmen : institutioneller Druck, gezielter Druck, Partnerschaften. Nach dieser Hypothese schaffen Demonstrationen gegen die Globalisierung und die Berichterstattung in den Medien ein Klima, das Initiativen im Bereich der sozialen Unternehmensverantwortung und vielleicht die Annahme von einschlägigen Regelungen begünstigt. 
Umgekehrt bergen solche Demonstrationen die Gefahr, durch die systematische Kritik und das Verteufeln der multinationalen Konzerne Energien der Bürger zu lähmen und den Blick auf den politischen Spielraum zwischen Rebellion und Resignation zu verstellen (siehe Schlussfolgerung).

\section{Gezielter Druck}

Auf Druckkampagnen der NGO folgen fast immer Partnerschaften zwischen dem betroffenen Unternehmen oder den NGO (den Moderatoren der Kampagne oder anderen). Dies bietet echte politische Chancen, vorausgesetzt, dass im Rahmen der Partnerschaft sinnvolle und relevante Initiativen ergriffen werden - was die Frage der Kontrolle (intern, extern, unabhängig?), der Anwendung und Beurteilung der freiwilligen Massnahmen aufwirft. Generell wurde beobachtet, dass die von NGO am stärksten kritisierten und herausgeforderten Unternehmen die umfassendste Sozialbilanz ausweisen. Das verdeutlicht gleichzeitig eine Grenze der Druckkampagnen: Kampagnen, welche Jahr für Jahr ein einziges Unternehmen - in der Regel einen Branchenführer - aufs Korn nehmen, drohen eine soziale Verantwortung der zwei Geschwindigkeiten zu begünstigen, wonach einige Musterschüler die Lektion aus der wiederholten Massregelung gelernt haben, der von NGO-Kritik verschonte Rest jedoch relativ unbemerkt ins Hintertreffen gerät. Insofern verdienen Kampagnen, welche eine Erweiterung des Zielpublikums anstreben, besonderes Interesse.

\section{$\square$ Partnerschaft mit Schwerpunkt auf der Unternehmenstätigkeit}

Die Unternehmen zeigen immer grösseres Interesse an Partnerschaften mit NGO, da solche Schritte einen wertvollen Image-Transfer erlauben. Die NGO erhalten dabei die Möglichkeit, ihr Fachwissen einzubringen und von innen an der Änderung der Unternehmenspraktiken mitzuwirken, was grundsätzlich interessante politische Chancen erschliesst. Die Frage einer Partnerschaft mit einem Unternehmen und die oft damit verbundene Gefahr, an Unabhängigkeit einzubüssen, werden von den verschiedenen NGO («politische» NGO, die den Schwerpunkt auf die Sensibilisierung der Öffentlichkeit und auf das Lobbying legen, oder aber «NGO vor Ort», die sich auf die Finanzierung konkreter Projekte konzentrieren) unterschiedlich beurteilt. Für Organisationen, die an beiden Fronten kämpfen, ist die Frage der Zweckmässigkeit von Partnerschaften mit dem Privatsektor besonders heikel. Nichtsdestotrotz haben zahlreiche Partnerschaften zwischen NGO und Unternehmen zu einer Verbesserung der sozialen oder ökologischen Auswirkungen eines Unternehmens geführt. Ein Helvetas-Vertreter kommentierte dazu, dass Helvetas Partnerschaften mit Unternehmen aufgeschlossen gegenüberstünde; man müsse mit den multinationalen Konzernen zusammenarbeiten und ihnen helfen, ihren Beitrag an die Entwicklung zu verbessern ${ }^{40}$.

\section{$\square$ Partnerschaft mit Schwerpunkt auf der Tätigkeit der NGO}

Sponsoring- und Marketingoperationen im Interesse einer Sache, die Unternehmen und NGO verbindet, erfahren seit mehreren Jahren steigenden Zuspruch, vor allem seitens der «NGO vor Ort», bisweilen aber auch seitens der «politischen NGO». Politisches Potenzial bergen hier das Ausmass und der Nutzen

40. Gespräch mit Tobias Meier, Verkaufsdirektor, Helvetas, 9. Oktober 2001. 
der Ressourcen, die das Unternehmen durch das Sponsoring oder Marketing für ein Anliegen für Sozialprojekte aufwendet. So beschlossen z.B. Pharmaunternehmen umfassende Arzneimittelschenkungen, und Informatikriesen engagierten sich in bedeutendem Umfang in Programmen zur Armutsverringerung. Praktisch alle Unternehmen verfügen über unterschiedlich entwickelte Wohltätigkeitsprogramme, über die mehr oder weniger kommuniziert wird.

\section{$\square$ Indirekte Evaluation}

Der Finanzsektor nimmt es zur Kenntnis, wenn NGO eine Kritikkampagne gegen ein Unternehmen starten oder im Rahmen einer Partnerschaft Initiativen unterstützen. Insofern nehmen die NGO nicht nur Einfluss auf die Beurteilung der Sozialperformance des Unternehmens und mithin auf die Gewichtung der Wertpapiere, welche die Erträge der - das Kriterium Nachhaltigkeit einbeziehenden - Investitionen ausmachen, sondern auch auf die Analyse und das Management der Goodwill-Risiken. Die NGO beeinflussen Unternehmen in zweifacher Hinsicht: Direkter und kurzfristiger Einfluss der Kampagnen / Partnerschaften und indirekter langfristiger Einfluss über die Sensibilisierung der Öffentlichkeit, die Informationsvermittlung in den Medien, die Wahrnehmung der Verbraucher und über bestimmte Investitionsstrategien. Die NGO, denen die öffentliche Meinung und die Medien wachsende Sympathie und Gehör schenken, und andere Akteure der Entwicklung sollten diese Entwicklungen und die entsprechenden politischen Chancen sorgfältig prüfen.

\section{$\square$ Direkte Evaluation}

Bestimmte NGO tragen direkt und weit gehend zur Evaluation der Sozialperformance des Unternehmens bei, indem sie Datenbanken und Notierungsstellen für Investoren aufbauen. Die NGO (und Regierungen), die sich für Entwicklung, Menschenrechte, Frieden oder Umweltschutz einsetzen, können die Evaluation der Sozialperformance eines Unternehmens bereichern, ihre Anliegen ergänzen und damit die Geltungskraft der Evaluation erhöhen. Als in der Zivilgesellschaft verankerte Organisationen können sie die Bürger (wie auch die Medien, Parteien, Gewerkschaften und Pensionskassen) überzeugen, die Schalthebel ethisches Konsumverhalten und nachhaltige Investitionen zu betätigen. Dazu arbeiten bestimmte NGO mit Auswertungsmechanismen, mit Zertifizierung oder mit Labels, denen sie einen Teil ihres Images ausleihen, zusammen. Dieser Bereich birgt zusätzliche Möglichkeiten zu den institutionellen Abläufen und sollte weiter untersucht werden.

\section{SCHLUSSFOLGERUNG: POLITISCHE MÖGLICHKEITEN WAHRNEHMEN}

Aus der Studie zu rund zwölf sozio-ökonomischen Themen mit globaler Tragweite geht hervor, dass direkte Druckkampagnen die Unternehmen zu Partnerschaften mit den NGO anspornen, um ihre Sozialperformance zu verbessern. Generell lässt die Analyse der Sozialbilanz der 28 Titel des Swiss Market Index ersehen, dass die von NGO am stärksten kritisierten und herausgeforderten Unternehmen die umfassendste Sozialbilanz ausweisen. Das spricht von der Macht, welche die NGO in der Vergangenheit über die multinationalen Konzerne ausübten, und deutet die politischen Möglichkeiten der Zukunft an. Es 
existieren drei Arten von Beziehungen zwischen NGO und Unternehmen und eine entsprechende Anzahl Rollen: Druck (kritische Rolle), Partnerschaft (unterstützende Rolle), Evaluation (beratende Rolle). Die Aktionen der NGO fallen in den Analysen des Finanzsektors immer stärker ins Gewicht, während die NGO ein neues politisches Aktionsfeld entdecken : ethisches Konsumverhalten, Evaluation der Sozialperformance des Unternehmens, nachhaltige Investition. Strategien, die auf gesetzlichen Zwang setzen, reichen nicht aus, um die Unternehmen zu einem verstärkten Engagement im Bereich Menschenrechte und Entwicklung zu bewegen. Politische Chancen bietet auch die direkte Beziehung zu den Unternehmen, ein differenzierter Druck also, womöglich kombiniert mit Verhandlungen, Partnerschaft, Evaluation und unter Bezugnahme auf ökonomische Grössen wie Wettbewerb, Profitstreben, Markenwert und Public Relations (vgl. Luhmann, 1989).

Die systematische Kritik an den multinationalen Unternehmen wendet sich gegen den eigentlich verfolgten Zweck und droht unbewusst das Potenzial der Bürger, sich als Beschäftigte, Kunden oder Aktionäre dieser Unternehmen zu engagieren, zu hemmen bzw. sich selbst und die Bürger davon abzubringen und sich selbst von der res publica $\mathrm{zu}$ entfernen. Motivierender wirkt folgende realistische Feststellung: Die Unternehmen tragen zur menschlichen Entwicklung bei ; bisweilen behindern sie sie aber; und vor allem könnten sie noch stärker dazu beitragen. Unternehmen sind wie die Menschen, aus denen sie bestehen: unvollkommen und fähig, sich zu ändern. Wichtig sind daher die differenzierte Wahrnehmung der ökonomischen Tätigkeit, Information, Evaluation und Stimulation. Dies setzt voraus, sich psychologisch in die multinationalen Konzerne hineinversetzen zu können, sich mit ihrem Auftreten vertraut zu machen, sie aufmerksam und individuell zu beobachten, mit ihnen in Kontakt zu treten und sie als ernst zu nehmende Akteure wahrzunehmen.

Der heutige Bürger wird als unpolitisch, individualistisch und aufs Private zurückgezogen charakterisiert. Passivität, das Aufgehen in der Masse, ist die Norm, ob der Bürger die multinationalen Konzerne liebt oder hasst. Werte wie soziale Gerechtigkeit, Solidarität, Brüderlichkeit werden aber nicht ignoriert oder abgelehnt. Diese Werte äussern sich in Abstimmungen, Demonstrationen und im Alltagsverhalten in der Familie und im informellen Kreis. Das mag vielleicht nicht genügen; vielleicht sind die Bürger enttäuscht und möchten mehr bzw. etwas anderes tun; vielleicht misstrauen sie militanten Zwangsjacken und beklagen das fehlende Engagement - weil sie denken, dass die Politik dort beginnt, wo ihre Freiheit aufhört, oder weil ihnen ganz einfach die Zeit fehlt. Diese Bürger sollten die Möglichkeit erhalten, mit politisch wirksamen und ihrer Lebensweise entsprechenden Mitteln zu handeln, die Schalthebel des sozialen Umbruchs zu betätigen, ohne dass ihr Komfort darunter leidet - kurz, man sollte ihnen benutzerfreundliche Politikprogramme anbieten. Ethisches Konsumverhalten, die Beurteilung der Sozialperformance des Unternehmens und nachhaltige Investition stecken zwar erst in den Kinderschuhen, gehören aber zu solchen Massnahmen. Es würde sich für Regierungen, Universitäten und NGO lohnen, Aufmerksamkeit und Ressourcen aufzuwenden, um die Mechanismen zu stärken, ihre weitere Entwicklung mitzugestalten und vielleicht den wichtigsten Mechanismen einen Teil ihrer Legitimität zu übertragen, da ein Potenzial zur Erweiterung des demokratischen Handelns besteht. 


\section{BIBLIOGRAPHIE}

Avery, Christopher L. (2000), Business and Human Rights in a time of change, Amnesty International UK, 2000.

Bendell, Jem (2000), Terms for Endearment - Business, NGOs and Sustainable Development, Greenleaf Publishing Limited in association with The New Academy of Business, 2000.

Elkington, John and Fennell, Shelly (1998), «Partners for Sustainability », Greener Management International, Issue 24, 1998.

Joerchel, Bastienne (1997), «Les organisations de protection de l'environnement et le secteur économique: de la confrontation à la coopération », in Martin Benninghoff, Bastienne Joerchel, Peter Knoepfel (dir.), L'écobusiness: enjeux et perspectives pour la politique de l'environnement, Helbing \& Lichtenhahn, Basel und Frankfurt am Main, 1997.

Luhmann, Niklas (1989), Ecological Communication, Cambridge University Press.

Mach, Antoine (2001), Entreprises suisses et droits de l'homme: confrontations et partenariats avec les $O N G$, Editions universitaires de Fribourg - Antenna Internationale, Institut interdisciplinaire d'éthique et des droits de l'homme, <www.unifr.ch/iiedh/publications/series/serie_droits.html>.

Teubner, Gunther (1997), Global Law without a State, Aldershot: Dartmouth Gower.

Utting, Peter (2001), «Verantwortung der Unternehmen für die nachhaltige Entwicklung», Jahrbuch Schweiz - Dritte Welt 2001, Universitätsinstitut für Entwicklungsstudien, Genf. 